\title{
On probabilistic categorization: The Markovian observer
}

\author{
LAWRENCE M. WARD \\ University of British Columbia, Vancouver, British Columbia, Canada \\ JAMES W. LIVINGSTON, JR. \\ Measurex, Inc., San Jose, California \\ and \\ JOSEPH LI \\ University of British Columbia, Vancouver, British Columbia, Canada
}

\begin{abstract}
A normative model (the Markovian observer) is described for a numerical decision task (an analogue of signal detection) in which the sequence of stimuli instantiates a two-state Markov chain. The expected-value observer of classical signal detection theory is a special case of the Markovian observer. An experiment is also described in which subjects performed the numerical detection task for different Markov chains of stimuli. Neither the ordinary expected-value observer nor the Markovian observer adequately described the performance of actual subjects, particularly when the stimulus sequence was random. A modification of the Markovian observer (the Markovian observer "ignorant" of the transition probabilities governing the stimulus sequence), similar to Kubovy and Healy's ideal-learner model, was simulated on a computer and provided a good qualitative fit to the data, especially to the distribution of violations of signal detection theory's single-criterion rule.
\end{abstract}

Probabilistic categorization involves deciding how to label a stimulus that may have come from one of several different sources with different probabilities. Since the situation is ubiquitous, it has been studied for a long time. Many models have been proposed for specific situations, such as signal detection theory (Green \& Swets, 1974), Thurstone's law of comparative judgment (Thurstone, 1927), and their more recent modifications (e.g., Kubovy \& Healy, 1977). These models have successfully described the average results in a useful way, and have provided a powerful basis for further psychophysical theory. Moreover, they have been extended into a wide variety of domains, such as memory and pain perception, where they have been useful in untangling motivational and response bias phenomena from more stimulus- and sensory-system-induced phenomena.

However, although hugely successful, such models are now acknowledged to be inadequate to describe the details of individuals' decision behavior. In particular, the

This research was supported by grants from the Natural Sciences and Engineering Research Council of Canada to L.M.W. and the Rutgers University Research Council to J.W.L. We thank Douglas Bobko for running the subjects. We are indebted to the reviewers for their useful critical comments, especially for detecting errors in the derivations of Equations 11 and 12 . Send reprint requests to Lawrence $M$. Ward, Department of Psychology, University of British Columbia, 2136 West Mall, Vancouver, BC V6T 1Y7, Canada. assumption of a single, fixed criterion has been questioned. Such phenomena as changes in sensitivity (e.g., $d^{\prime}$ in signal detection theory) independent of changes in signal strength, sequential dependencies, directional sensory generalization, and changes in slopes of receiver operating characteristic (ROC) curves as a function of signal strength all violate this assumption (cf. Kubovy \& Healy, 1977; Larkin, 1971; Treisman \& Williams, 1984). Moreover, in numerical detection situations designed to study binary decision behavior independent of sensory system properties, not only do subjects fail to use a singlecriterion decision rule (Kubovy, Rapoport, \& Tversky, 1971; Ward, 1973), but they also actually report changing their criterion from trial to trial (Kubovy \& Healy, 1977). Kubovy and Healy (1977) review a number of alternatives to the standard Bayesian expected-value observer developed in signal detection theory. These include various additive operator models in which the single criterion of signal detection theory is moved around with different probabilities in case of an error or a correct response, because of drift of its memory representation, or because of the gambler's fallacy. These models, and various special cases of them, can usually be rejected on the basis of subjects' reports of where they move their criteria. Likewise, various probabilistic models, which have no criteria at all, such as Lee's (1971) micromatching model and Schoeffler's (1965) directional stimulus generalization model, can be rejected, at least for numer- 
ical detection, by the data of Kubovy et al. (1971), Kubovy and Healy (1977), and Ward (1973).

As an alternative to all of these models, Kubovy and Healy (1977) proposed their "ideal-learner" form of a dynamic criterion model. They assumed that a subject in a signal detection situation might know that two probability distributions, with equal variances and unknown means, were being sampled. Subjects must estimate the means from the sequence of stimuli. If the subject's response criterion is based upon these estimates, which will vary from trial to trial, then the criterion will also vary from trial to trial, in a predictable fashion. Criterion shifts observed in a numerical detection situation by Kubovy and Healy (1977) generally supported this ideallearner model, while rejecting other dynamic criterion models and Schoeffler's probabilistic model. Unfortunately, the observed shift of criteria after correct responses was not always as predicted by the ideal-learner model, a fact that was explained by invoking the gambler's fallacy for shifts in the "wrong" direction. Although such a strategy is plausible, subjects violate the single-criterion rule in numerical detection tasks even when they know the means of both probability distributions (Ward, 1973). It is possible that at least some shifting of criteria in such situations arises for other reasons.

Ward (1973) suggested that in such situations subjects' decisions were influenced by sequential response biases given rise to by their attempts to use sequential information to aid their decisions. When stimuli are presented repetitiously on a series of trials, subjects are justified a priori in searching for information in the sequence. Particularly in difficult and ambiguous decision situations, such as signal detection, such a tendency could be exaggerated. Evidence from a number of studies suggests that humans are highly sensitive to such sequential information, even to the extent that they manufacture it for themselves when it is not objectively present. Perhaps the most direct evidence of this comes from the probability learning literature (e.g., Estes, 1972). Anderson (1960) and others (e.g., Restle, 1966; Rose \& Vitz, 1966; Vitz \& Todd, 1967) have shown, in the binary prediction setting, for example, that subjects distribute their responses so as to reflect the presence of at least first-order sequential dependencies in the sequence of stimuli. When first-order sequential dependencies are indeed present in the stimulus sequence, subjects can use them to improve probabilistic categorization performance, but they actually do more poorly than they should when such dependencies are absent (Ward, 1973). They appear to be acting as if dependencies were present, which causes them to violate the ideal observer's single-criterion decision rule in that situation.

In what follows, we first develop a Bayesian expectedvalue observer model of decision performance, the "Markovian observer," for a generalized probabilistic categorization situation, and evaluate its ability to explain performance in a numerical detection experiment in some conditions of which first-order sequential dependencies occur in the sequence of stimuli. The Markovian observer is intended to be a normative model that contains the Bayesian expected-value form of the ideal observer from signal detection theory as a special case. The intent here is to determine whether the normative model is sufficient to explain subjects' behavior in the numerical detection situation. It is shown (by computer simulation) that an extension of the normative model, called "the ignorant Markovian observer," is sufficient to explain violations of the single-criterion decision rule, especially when no stimulus sequential dependencies are present. It is also argued that a nonnormative form of the extended model may be required to explain the response latencies observed in the experiment. Finally, possible generalizations of the ignorant Markovian observer model are described that would be needed to account for other categorization phenomena, such as directional stimulus generalization, and behavior in other situations, such as with sensory stimuli, or without feedback.

\section{THE MARKOVIAN OBSERVER}

Consider a probabilistic categorization situation in which there exist two probability distributions over the same random variable, $X$, labeled " 1 " and " 2 ," from which samples, $X_{n}$, are drawn according to the transition matrix of a first-order Markov chain,

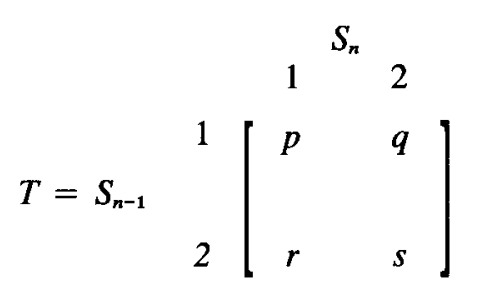

where $p, q, r, s$ are the (conditional) transition probabilities, $p+q=1, r+s=1$, and $S_{n}$ and $S_{n-1}$ are states (indicating from which distribution a sample is drawn) on trials $n$ and $n-1$, respectively. We assume an initial state vector, $\langle 0.5,0.5\rangle$. This results in Markov chains of samples from the two distributions ("stimuli") in which the asymptotic state probabilities are

$$
\begin{aligned}
& P\left(S_{n}=1\right)=r /(r+q), \\
& P\left(S_{n}=2\right)=q /(r+q) .
\end{aligned}
$$

An "observer" in this situation is required to give responses $\left(\boldsymbol{R}_{n}\right)$ " 1 ", or " 2 "' (assertions that the sample on Trial $n, X_{n}$, came from distribution " 1 " or " 2 ") and is rewarded according to a payoff matrix

$$
\begin{aligned}
& R_{n} \\
& P M=S_{n}\left[\begin{array}{cc}
1 & 2 \\
V_{11} & V_{12} \\
& \\
V_{21} & V_{22}
\end{array}\right]
\end{aligned}
$$


An ordinary expected-value observer from signal detection theory (see, e.g., Green \& Swets, 1974) would use the following decision rule to maximize the expected value of responses in this situation:

$$
\text { If } E\left(V \mid x_{n}, R_{n}=1\right) \quad\left\{\begin{array}{l}
> \\
\leq
\end{array}\right\} E\left(V \mid x_{n}, R_{n}=2\right) \text { say }\left\{\begin{array}{l}
1 \\
2
\end{array}\right\}
$$

where $E\left(V \mid x_{n}, R_{n}=1\right)$ is the expected value conditional on making $R_{n}=1$ to stimulus $x_{n}$, and where

$$
\begin{aligned}
E\left(V \mid x_{n}, R_{n}=1\right)= & {\left[P\left(S_{n}=1 \mid x_{n}\right) \cdot V_{11}\right] } \\
& -\left[P\left(S_{n}=2 \mid x_{n}\right) \cdot V_{21}\right] \\
E\left(V \mid x_{n}, R_{n}=2\right)= & {\left[P\left(S_{n}=2 \mid x_{n}\right) \cdot V_{22}\right] } \\
& -\left[P\left(S_{n}=1 \mid x_{n}\right) \cdot V_{12}\right]
\end{aligned}
$$

Using Bayes's theorem to obtain the conditional probabilities in Equations 5, and rearranging, the decision rule can be expressed in the usual way:

$$
\text { If } \lambda\left(x_{n}\right)\left\{\begin{array}{l}
< \\
\geq
\end{array}\right\} \quad \beta \text { say }\left\{\begin{array}{l}
1 \\
2
\end{array}\right\}
$$

where

$$
\begin{aligned}
\lambda\left(x_{n}\right) & =f\left(x_{n} \mid S_{n}=2\right) / f\left(x_{n} \mid S_{n}=1\right), \\
\beta & =\left[P\left(S_{n}=1\right) / P\left(S_{n}=2\right)\right] \cdot V_{p m},
\end{aligned}
$$

and $V_{p m}=\left(V_{11}+V_{12}\right) /\left(V_{22}+V_{21}\right)$. In Equation 7 , $f\left(x_{n} \mid S_{n}=2\right)$ and $f\left(x_{n} \mid S_{n}=1\right)$ are the probability density functions describing the probability distributions from which the stimulus samples are taken. It can be seen that for the usual expected-value observer of signal detection theory there is no provision for taking account of firstorder dependencies in the stimulus sequence. It is assumed that the stimulus sequence is governed by a Markov transition matrix with $p=r$ (and thus $q=s$ ) so that $P\left(S_{n}=1\right)$ and $P\left(S_{n}=2\right)$ can vary but there is no dependency of $S_{n}$ on $S_{n-1}$. This is the only situation in which expected value will be maximized by the decision rule described by Equation 6.

The (more general) Markovian expected-value observer is created by replacing the asymptotic state probabilities in the expressions above with the elements of the transition matrix governing the sequence of samples from the two probability distributions, that is, $p, q, r$, and $s$, and making the decision rule conditional on $S_{n-1}$ as follows:

$$
S_{n-1}=1: \quad \text { if } \lambda\left(x_{n}\right)\left\{\begin{array}{c}
< \\
\geq
\end{array}\right\} \beta_{1} \text { say }\left\{\begin{array}{l}
1 \\
2
\end{array}\right\}
$$

where $\beta_{1}=(p / q) \cdot V_{p m}$, and

$$
S_{n-1}=2: \quad \text { if } \lambda\left(x_{n}\right)\left\{\begin{array}{c}
< \\
\geq
\end{array}\right\} \beta_{2} \text { say }\left\{\begin{array}{l}
1 \\
2
\end{array}\right\}
$$

where $\beta_{2}=(r / s) \cdot V_{p m}$. Notice that the Markovian observer uses two cutoff points; which one is used on a particular trial is conditional on $S_{n-1}$. The Markovian observer will perform identically to the usual expected-value observer when $r=p$ (and thus $q=s$ ) because then $r+q$ $=p+s=1$, which implies that $P\left(S_{n}=1\right)=p=r$ and $P\left(S_{n}=2\right)=q=s$, so that $\beta_{1}=\beta_{2}=\beta$. However, it will be sensitive to first-order dependencies in the stimulus sequence if that condition does not obtain.

If $\lambda(x)$ is a monotonic function of $x$, as it is, for example, when $f\left(x_{n} \mid S_{n}=1\right)$ and $f\left(x_{n} \mid S_{n}=2\right)$ are Gaussian, then we can describe decision rules and theoretical values of $P(H)$ (the probability of a hit) and $P(F A)$ (the probability of a false alarm) in terms of arbitrary values of the random variable $X$, for example, $x_{i}$. The remainder of the discussion will be cast in these terms for convenience. Also, in what follows, it is assumed that $E\left(X_{n} \mid S_{n}=1\right)<$ $E\left(X_{n} \mid S_{n}=2\right)$. A parallel development is possible for the case where $E\left(X_{n} \mid S_{n}=1\right)>E\left(X_{n} \mid S_{n}=2\right)$.

To describe the ROC curve, conditional on $S_{n-1}$, for the Markovian observer, we need expressions for the probabilities of hits and false alarms for each state of $S_{n-1}$. These are:

$$
S_{n-1}=1: P_{1}(H)=1-F_{2}\left(x_{1}\right) ; P_{1}(F A)=1-F_{1}\left(x_{1}\right),
$$

$$
S_{n-1}=2: P_{2}(H)=1-F_{2}\left(x_{2}\right) ; P_{2}(F A)=1-F_{1}\left(x_{2}\right) \text {, }
$$

where $\lambda\left(x_{1}\right)=\beta_{1}$ and $\lambda\left(x_{2}\right)=\beta_{2}$, and $F_{1}$ and $F_{2}$ are the cumulative distribution functions corresponding to the density functions $f\left(x_{n} \mid S_{n}=1\right)$ and $f\left(x_{n} \mid S_{n}=2\right)$, respectively. Equations 10 would generate pairs of points on conditional ROC curves created by varying, for example, $T$ or $P M$. Since they depend only on signal strength, all conditional $d^{\prime}$ estimates should be roughly equal, and usually they are for human observers (e.g., Sandusky, 1971; Ward, 1973).

To evaluate the Markovian observer relative to the usual, non-Markovian, expected-value observer of signal detection theory, it is necessary to have expressions for unconditional probabilities of hits and false alarms. To do this, consider an unconditional hit-and-false-alarm matrix. Whenever $x_{1}<x_{2}$, the unconditional probability of a hit, $P_{m}(H)$, will be

$$
\begin{aligned}
P_{m}(H)= & P\left(R_{n}=2 \mid S_{n}=2\right) \\
= & P\left(X_{n} \geq x_{2} \mid S_{n}=2\right) \\
& +P\left(x_{1}<X_{n}<x_{2} \text { and } S_{n-1}=1 \mid S_{n}=2\right) \\
= & P\left(X_{n} \geq x_{2} \mid S_{n}=2\right)+P\left(x_{1}<X_{n}<x_{2} \mid S_{n}=2\right) \\
& \cdot P\left(S_{n-1}=1 \mid S_{n}=2 \text { and } x_{1}<X_{n}<x_{2}\right)
\end{aligned}
$$

Since $P\left(S_{n-1}=1 \mid S_{n}=2\right.$ and $\left.x_{1}<X_{n}<x_{2}\right)=$ $P\left(S_{n-1}=1 \mid S_{n}=2\right)=P\left(S_{n-1}=1\right.$ and $\left.S_{n}=2\right) / P\left(S_{n}=2\right)=$ 
$P\left(S_{n}=2 \mid S_{n-1}=1\right) \cdot P\left(S_{n-1}=1\right) / P\left(S_{n}=2\right)=$ $q[r /(r+q)] /[q /(r+q)]=r$, this can be written

$$
P_{m}(H)=\left[1-F_{2}\left(x_{2}\right)\right]+r \cdot\left[F_{2}\left(x_{2}\right)-F_{2}\left(x_{1}\right)\right] .
$$

Similarly, the unconditional probability of a false alarm, $P_{m}(F A)$, will be

$$
\begin{aligned}
P_{m}(F A)= & P\left(R_{n}=2 \mid S_{n}=1\right) \\
= & P\left(X_{n} \geq x_{2} \mid S_{n}=1\right) \\
& +P\left(x_{1}<X_{n}<x_{2} \text { and } S_{n-1}=1 \mid S_{n}=1\right) \\
= & P\left(X_{n} \geq x_{2} \mid S_{n}=1\right)+P\left(x_{1}<X_{n}<x_{2} \mid S_{n}=1\right) \\
& \cdot P\left(S_{n-1}=1 \mid S_{n}=1 \text { and } x_{1}<X_{n}<x_{2}\right)
\end{aligned}
$$

Again, since $P\left(S_{n-1}=1 \mid S_{n}=1\right.$ and $\left.x_{1}<X_{n}<x_{2}\right)=$ $P\left(S_{n-1}=1 \mid S_{n}=1\right)=P\left(S_{n-1}=1\right.$ and $\left.S_{n}=1\right) / P\left(S_{n}=1\right)$ $=P\left(S_{n}=1 \mid S_{n}=1\right) \cdot P\left(S_{n-1}=1\right) / P\left(S_{n}=1\right)=$ $p[r /(r+q)] /[r /(r+q)]=p$, the above can be written

$$
P_{m}(F A)=\left[1-F_{1}\left(x_{2}\right)\right]+p \cdot\left[F_{1}\left(x_{2}\right)-F_{1}\left(x_{1}\right)\right] .
$$

The corresponding equations for the case where $x_{2}<x_{1}$ are:

$$
\begin{aligned}
& P_{m}(H)=\left[1-F_{2}\left(x_{1}\right)\right]+s \cdot\left[F_{2}\left(x_{1}\right)-F_{2}\left(x_{2}\right)\right], \\
& P_{m}(F A)=\left[1-F_{1}\left(x_{1}\right)\right]+q \cdot\left[F_{1}\left(x_{1}\right)-F_{1}\left(x_{2}\right)\right] .
\end{aligned}
$$

Now the probability of a hit and of a false alarm for the usual expected-value observer are (when $x_{1}<x_{c}<x_{2}$ )

$$
P_{e v}(H)=1-F_{2}\left(x_{c}\right)=\left[1-F_{2}\left(x_{2}\right)\right]+\left[F_{2}\left(x_{2}\right)-F_{2}\left(x_{c}\right)\right]
$$

and

$$
P_{e v}(F A)=1-F_{1}\left(x_{c}\right)=\left[1-F_{1}\left(x_{2}\right)\right]+\left[F_{1}\left(x_{2}\right)-F_{1}\left(x_{c}\right)\right],
$$

respectively, where $\lambda\left(x_{c}\right)=\beta=\left[P\left(S_{n}=1\right) / P\left(S_{n}=2\right)\right]$ - $V_{p m}$; in other words, the single criterion of the expectedvalue observer. Clearly, when $p=r$ (and thus $q=s$ ), $x_{1}$ $=x_{2}=x_{c}, P_{m}(H)=P_{e v}(H)$, and $P_{m}(F A)=P_{e v}(F A)$. However, when this is not the case, $P_{m}(H)>P_{e v}(H)$ and $P_{m}(F A)<P_{e v}(F A)$, and so $d_{m}^{\prime}>d^{\prime}{ }_{e v}$. This is demonstrated by the simulations discussed later.

Another useful quantity to calculate for the Markovian observer is the probability of violating the expected-value observer's single-criterion decision rule. Since, whenever $p=r$ does not hold, the Markovian observer will use either $x_{1}$ or $x_{2}$ as criterion values, instead of $x_{c}$, its decisions will disagree with the expected-value observer's decision whenever $x_{n}$ falls between the criterion in use and $x_{c}$. More formally, the probability of a violation of the expectedvalue observer's decision rule on trial $n, P_{v n}$, is

$P_{v n}=P\left[\left(X_{n}<x_{c}\right.\right.$ and $\left.R_{n}=2\right)$ or $\left(X_{n} \geq x_{c}\right.$ and $\left.\left.R_{n}=1\right)\right]$.
Consider the case where $x_{1}<x_{2}$. Here we have

$$
\begin{aligned}
P_{v 1}= & P\left(\text { violation } \mid S_{n-1}=1\right) \\
= & P\left(S_{n}=1 \mid S_{n-1}=1\right) \cdot P\left(x_{1}<X_{n}<x_{c} \mid S_{n}=1\right) \\
& +P\left(S_{n}=2 \mid S_{n-1}=1\right) \cdot P\left(x_{1}<X_{n}<x_{c} \mid S_{n}=2\right) \\
= & p \cdot\left[F_{1}\left(x_{c}\right)-F_{1}\left(x_{1}\right)\right]+q \cdot\left[F_{2}\left(x_{c}\right)^{\prime}-F_{2}\left(x_{1}\right)\right]
\end{aligned}
$$

and

$$
\begin{aligned}
P_{v 2}= & P\left(\text { violation } \mid S_{n-1}=2\right) \\
= & P\left(S_{n}=1 \mid S_{n-1}=2\right) \cdot P\left(x_{c}<X_{n}<x_{2} \mid S_{n}=1\right) \\
& +P\left(S_{n}=2 \mid S_{n-1}=2\right) \cdot P\left(x_{c}<X_{n}<x_{2} \mid S_{n}=2\right) \\
= & r \cdot\left[F_{1}\left(x_{2}\right)-F_{1}\left(x_{c}\right)\right]+s \cdot\left[F_{2}\left(x_{2}\right)-F_{2}\left(x_{c}\right)\right] .
\end{aligned}
$$

The unconditional probability of a violation, $P_{v}$, is then

$$
P_{v}=P\left(S_{n-1}=1\right) \cdot P_{v 1}+P\left(S_{n-1}=2\right) \cdot P_{v 2}
$$

or

$$
\begin{aligned}
P_{v}= & {[r /(r+q)] \cdot\left\{p \cdot\left[F_{1}\left(x_{c}\right)-F_{1}\left(x_{1}\right)\right]\right.} \\
& \left.+q \cdot\left[F_{2}\left(x_{c}\right)-F_{2}\left(x_{1}\right)\right]\right\} \\
& +[q /(r+q)] \cdot\left\{r \cdot\left[F_{1}\left(x_{2}\right)-F_{1}\left(x_{c}\right)\right]\right. \\
& \left.+s \cdot\left[F_{2}\left(x_{2}\right)-F_{2}\left(x_{c}\right)\right]\right\} .
\end{aligned}
$$

The corresponding equation for $P_{v}$ when $x_{2}<x_{1}$ is

$$
\begin{aligned}
P_{v}= & {[r /(r+q)] \cdot\left\{p \cdot\left[F_{1}\left(x_{1}\right)-F_{1}\left(x_{c}\right)\right]\right.} \\
& \left.+q \cdot\left[F_{2}\left(x_{1}\right)-F_{2}\left(x_{c}\right)\right]\right\} \\
& +[q /(r+q)] \cdot\left\{r \cdot\left[F_{1}\left(x_{c}\right)-F_{1}\left(x_{2}\right)\right]\right. \\
& \left.+s \cdot\left[F_{2}\left(x_{c}\right)-F_{2}\left(x_{2}\right)\right]\right\} .
\end{aligned}
$$

Notice that whenever $p=r, x_{1}=x_{2}=x_{c}$ and $P_{v}=0$.

The number and proportion of such violations made by subjects were reported by both Kubovy et al. (1971) and Ward (1973), and they provide an important index of the extent to which subjects are following either model. Also, the distribution of such violations over the likelihood ratio axis is of interest. This will be estimated later from computer simulations of the Markovian observer and used as a criterion by which to evaluate the success of the models.

A few examples might help to clarify the operation of the model of the Markovian observer in comparison with the expected-value observer. Table 1 lists the various relevant quantities for seven examples of different decision situations. In all of them, samples are drawn from Gaussian distributions $\left[f\left(x_{n} \mid S_{n}=1\right)\right.$ and $\left.f\left(x_{n} \mid S_{n}=2\right)\right]$ with $\sigma_{1}=\sigma_{2}=1, \mu_{1}=0$, and $\mu_{2}=1$. Examples 1 through 5 differ only in the transition matrix that governs the sequence of samples from the two probability distributions; Example 6 is the same as Example 1, except that $V_{p m}=2.33$; Example 7 is the same as Example 3, except that $V_{p m}=$ 1.5. Unconditional probabilities of hits and false alarms 
Table 1

Examples Comparing SDT with the Markovian Observer

\begin{tabular}{|c|c|c|c|c|c|c|c|c|c|c|}
\hline Example & $T$ & & $V_{p m}$ & $\beta_{1}$ & $\beta_{2}$ & $\beta$ & $P_{m}(H)$ & $P_{m}(F A)$ & $d_{m}^{\prime}$ & $P_{v}$ \\
\hline 1 & $\begin{array}{l}.5 \\
.5\end{array}$ & $\begin{array}{l}.5 \\
.5\end{array}$ & 1.00 & 1.00 & 1.00 & 1.00 & .690 & .310 & 1.00 & .000 \\
\hline 2 & $\begin{array}{l}.9 \\
.9\end{array}$ & $\begin{array}{l}.1 \\
.1\end{array}$ & 1.00 & 9.00 & 9.00 & 9.00 & .045 & .003 & 1.00 & .000 \\
\hline 3 & $\begin{array}{l}.9 \\
.1\end{array}$ & $\begin{array}{l}.1 \\
.9\end{array}$ & 1.00 & 9.00 & 0.11 & 1.00 & .902 & .098 & 2.56 & .340 \\
\hline 4 & $\begin{array}{l}.1 \\
.9\end{array}$ & $\begin{array}{l}.9 \\
.1\end{array}$ & 1.00 & 0.11 & 9.00 & 1.00 & .902 & .098 & 2.56 & .340 \\
\hline 5 & $\begin{array}{l}.7 \\
.1\end{array}$ & $\begin{array}{l}.3 \\
.9\end{array}$ & 1.00 & 2.33 & 0.11 & 0.33 & .933 & .349 & 2.28 & .207 \\
\hline 6 & $\begin{array}{l}.5 \\
.5\end{array}$ & $\begin{array}{l}.5 \\
.5\end{array}$ & 2.33 & 2.33 & 2.33 & 2.33 & .430 & .220 & 1.00 & .000 \\
\hline 7 & $\begin{array}{l}.9 \\
.1\end{array}$ & $\begin{array}{l}.1 \\
.9\end{array}$ & 1.50 & 13.5 & .167 & 1.50 & .883 & .091 & 2.56 & .340 \\
\hline
\end{tabular}

and $d^{\prime}$ 's listed in Table 1 are those for the Markovian observer. $d^{\prime}=1$ in all examples for the expected-value observer using the $\beta$ listed in the table. Unconditional probabilities of hits and false alarms are not listed for this observer, but are the same as in Example 1 for all other examples except for 2 and 6 , where they are the same as those listed in the table for the Markovian observer, 5 , where $P_{e v}(H)=0.95, P_{e v}(F A)=0.73$, and 7, where $P_{e v}(H)=0.54, P_{e v}(F A)=0.18$.

In what follows, we describe an experiment designed to provide data with which to compare the predictions of the Markovian observer, suggest modifications to that normative model in the spirit of the ideal-learner model of Kubovy and Healy, simulate several versions of the modified Markovian model, and finally suggest additional modifications needed to account for latency data, which are not addressed by any of the existing models.

\section{THE EXPERIMENT}

Subjects were asked to make probabilistic categorizations in the numerical detection situation originally described by Kubovy et al. (1971) and also used by Ward (1973). Subjects made judgments as to which of two approximately normal distributions on the integers was sampled on a given trial, given the integer sampled. As in Ward's study, stimulus sequences were governed by various transition matrices, some of which introduced sequential information. The present study extended that study to somewhat different transition matrices, using a withinsubject design in which all subjects performed in all conditions, and also allowed an analysis of response latencies in the various conditions.

\section{Method}

Subjects. The subjects were 5 Rutgers University undergraduate volunteers, all of whom were registered in an undergraduate statistics course in the Psychology Department at Livingston College (of Rutgers).

Design. Each state of the two-state Markov chain governing the sequence of stimuli corresponded to an integer sampled from one of two approximately normal distributions over the integers with means of $\mu_{1}=1,500$ and $\mu_{2}=1,600$ and standard deviations of $\sigma_{1}=\sigma_{2}=100$. On each trial, a subject was presented with an integer drawn from one of these two distributions and had to respond "1" if he or she thought it was drawn from distribution 1 (mean 1,500 ) and ' 2 "' if he or she thought it was from distribution 2 (mean $1,600)$. Five different transition matrices created five different conditions. Values of $p=s=0.1,0.2,0.5,0.8$, and 0.9 defined the five transition matrices. All subjects participated in two sessions of $500+$ trials under each transition matrix. Each subject received the conditions in a different order in a Latin square design. The subjects were paid only for performance, according to a payoff matrix in which a correct response (either 1 or 2) was accorded a payoff of 0.7 cent and there was no penalty for incorrect responding. Rewards were paid at the end of the experiment, but the subjects were told of the day's earnings at the end of each session. The subjects earned an average of $\$ 2.81$ for each roughly 45 -min session.

Apparatus. A PDP-11/40 computer controlled stimulus presentation, recorded responses and responses latencies, and provided feedback. Stimuli were presented on a video monitor, and responses were made by pressing either a left (1) or right (2) response button. Latency timing was accurate to $1 \mathrm{msec}$.

Procedure. At the first session, the subjects were provided with written instructions that characterized the task as one of judgment under conditions of uncertainty. The subjects were told the payoff conditions but were not told the exact amount of the payoff for each correct judgment. They were told that there were two generators of observations, processes 1 and 2, with "process 1 generat[ing] numbers around the value 1500 , while process 2 produces values around 1600." The subjects were not given explicit values for the 
standard deviations of the two processes, but were told that "both processes produce values that could have come from the other." Moderate accuracy set was encouraged by highlighting the availability of reward on each trial. After they had read the instructions, the subjects were given 20 practice trials and any remaining misunderstandings were dispelled by paraphrasing the printed instructions. At no time were the subjects told anything about the transition matrices that governed the sequence of stimuli.

At the beginning of each trial, the stimulus was displayed in the following format:

\section{OBSERVATION FOR TRIAL $n$ IS $x$}

on the center of the video monitor. After the subject pressed either the left or right button, feedback was displayed on the screen for $1 \mathrm{sec}$ in the following format:

\section{$\left\{\begin{array}{c}\text { Right } \\ \text { Wrong }\end{array}\right\}$ OBSERVATION WAS FROM PROCESS $i$}

Following this display, the screen was cleared for the 1.5-sec intertrial interval, after which the next stimulus was displayed.

\section{Results}

Sensitivity, $d^{\prime}$. Table 2 displays the average values of the sensitivity parameter of signal detection theory, $d^{\prime}$, for each of the subjects and conditions. The Latin square ANOVA yielded no significant effects for subjects or order of conditions $[F(4,12)=2.87, p=.07 ; F(4,12)$ $=1.28 ; p=.33$ ], although the $F$ for subjects does reflect the aberrant behavior of Subject 4 in the $p=s=0.5$ condition. On the other hand, the effect of condition is highly reliable $[F(4,12)=87.92, p \ll .0001]$. Average $d^{\prime}$ was about 2.5 when $p=s=0.9$ or $p=s=0.1$, about 1.6 or 1.7 when $p=s=0.8$ or $p=s=0.2$, but only about 0.7 when $p=s=0.5$. Leaving out Subject 4's data for that condition (a clearly aberrant result) leaves the $d^{\prime}$ for $p=s=0.5$ at only 0.84 , still considerably less than the nominal value of 1.00 for these conditions. These results replicate and extend those of Ward (1973). Subjects can use the information in the transition matrix governing the sequence of stimuli to improve their performance. However, when no such information is present, their performance is less than optimal.

Table 2 also displays predictions of performance in the various conditions from the Markovian observer model outlined above. Nominal predictions were obtained by inserting the operative parameter values and distribution function (Gaussian) in Equations 11 and 12 for unconditional probabilities of hits and false alarms, and calculating $d^{\prime}$ 's for the resulting values. Simulated predictions were obtained by applying the Markovian decision rule to the

Table 2

Sensitivity $\left(d^{\prime}\right)$ Scores and Predictions

\begin{tabular}{ccccccccc}
\hline$p=s$ & $S_{1}$ & $S_{2}$ & $S_{3}$ & \multicolumn{1}{c}{$S_{4}$} & $S_{5}$ & Mean & Nom & Sim \\
\hline 0.1 & 2.60 & 2.43 & 2.54 & 2.66 & 2.54 & 2.55 & 2.56 & 2.61 \\
0.2 & 1.73 & 1.31 & 1.27 & 1.18 & 1.63 & 1.42 & 1.78 & 1.81 \\
0.5 & 0.78 & 0.79 & 0.80 & -0.02 & 1.00 & 0.67 & 1.00 & 0.95 \\
0.8 & 1.48 & 1.52 & 1.35 & 1.49 & 1.74 & 1.51 & 1.78 & 1.71 \\
0.9 & 2.73 & 2.52 & 2.56 & 2.34 & 2.54 & 2.54 & 2.56 & 2.66 \\
\hline
\end{tabular}

Note-Nom $=$ nominal prediction using Equations 11 and $12 ; \mathrm{Sim}=$ simulated prediction.
Table 3

Proportion of Violations of Single-Criterion Rule, $P_{v}$

\begin{tabular}{ccccccccc}
\hline$p=s$ & $S_{1}$ & $S_{2}$ & $S_{3}$ & $S_{4}$ & $S_{5}$ & Mean & Nom & Sim \\
\hline 0.1 & .308 & .371 & .365 & .344 & .376 & .353 & .340 & .346 \\
0.2 & .376 & .121 & .256 & .410 & .374 & .308 & .324 & .322 \\
0.5 & .069 & .092 & .175 & .499 & .126 & .192 & .000 & .000 \\
0.8 & .271 & .360 & .242 & .389 & .328 & .318 & .324 & .325 \\
0.9 & .334 & .341 & .340 & .388 & .322 & .345 & .340 & .338 \\
\hline
\end{tabular}

Note-Nom $=$ nominal prediction from Equations $15 ;$ Sim $=$ simulated prediction.

sequence of stimuli actually received by each subject. Nominal and simulated predictions match closely. Both predictions are reasonably close to subjects' performance in the extreme conditions ( $p=s=0.9$ and $p=s=0.1$ ), but deviate considerably in the other conditions. Better performance by the Markovian observer when $p=s=0.8$ and $\mathrm{p}=s=0.2$ implies that subjects were not capitalizing on all available sequential information in those conditions. However, subjects' poorer performance when $p=s=0.5$, when the Markovian and the usual expected-value observer predictions are the same, implies that subjects may still have been using more than one decision criterion.

Proportion of violations of the single-criterion rule, $P_{v}$. Table 3 displays the proportion of violations of the single-criterion rule (employed by the usual expectedvalue observer) found in the data of the subjects in the various conditions. From Table 3 it can be seen that the subjects in this experiment regularly violated the singlecriterion rule, even when $p=s=0.5$, when that rule was the optimal one. Moreover, they violated the rule more, the more extreme the transition probabilities were. Again, while ANOVA revealed no significant subject or order effect $[F(4,12)=2.87, p=.07 ; F(4,12)=2.15$, $p=.14]$, there was a reliable effect of condition $[F(4,12)$ $=3.35, p<.05]$. The overall proportion of violations when $p=s=0.5$ was 0.19 (or 0.12 , if we drop Subject 4 , who violated the rule in that condition at least three times as much as any other subject). The corrected figure of 0.12 is about the result obtained by Ward (1973) and again higher than Kubovy et al.'s 0.06 .

Table 3 also displays predictions from the Markovian observer model for violations of the single-criterion rule. Normative predictions were calculated from Equations 15, using the appropriate parameter values for the various conditions. Simulated predictions were obtained by simulations similar to those run for $d^{\prime}$ on the actual sequence of stimuli presented to the subjects. Again, the simulations are in reasonable agreement. They do a good job of predicting $P_{v}$ in all but the middle condition. In that condition, of course, no violations are predicted by either of the models under consideration.

More evidence of the usefulness of the Markovian observer comes from a study of the distribution of violations of the single-criterion model for the various conditions. Figure 1 shows these distributions for the data (collapsed across subjects) of each condition, along with 

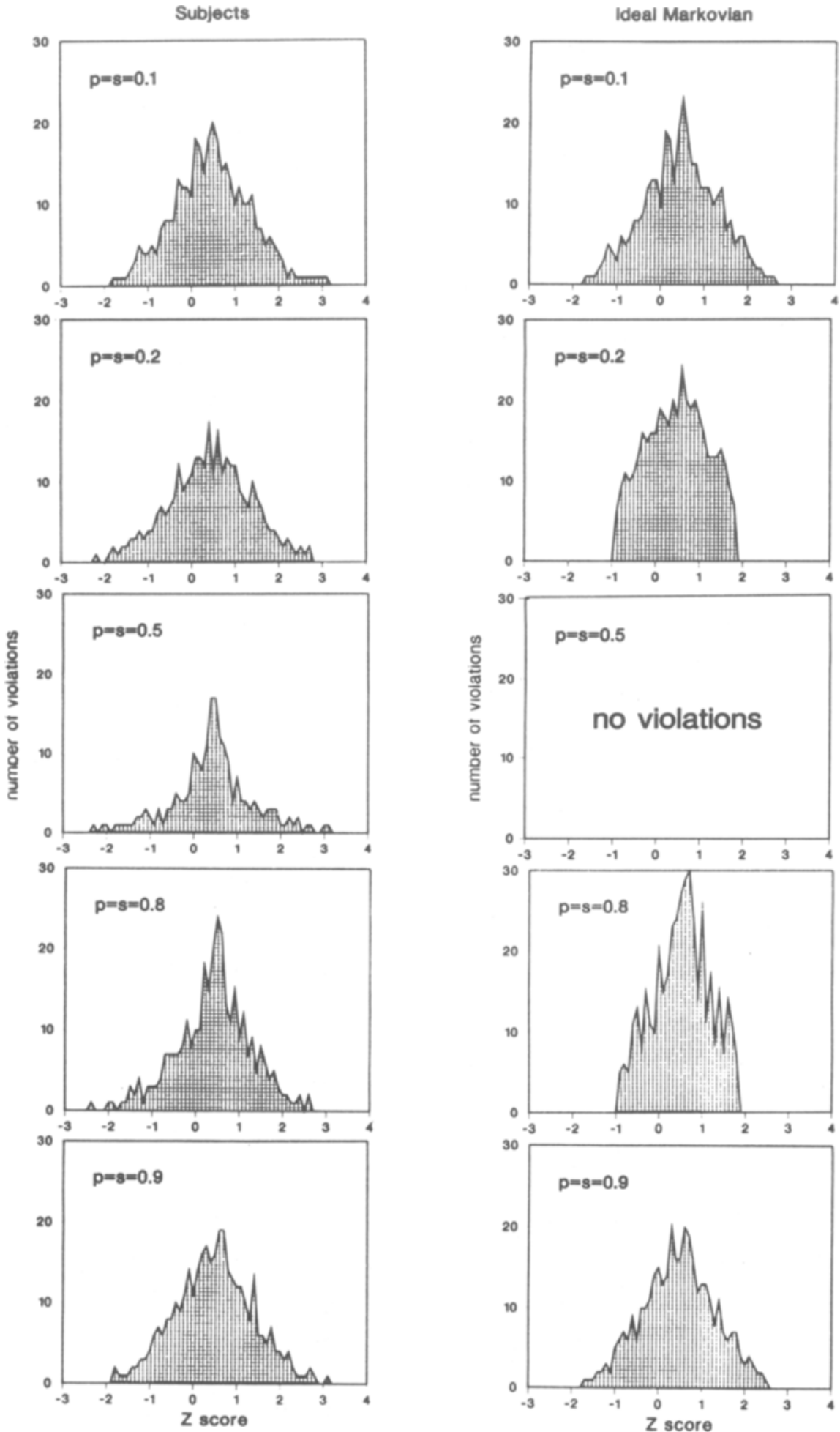

Figure 1. Distributions of violations of the single-criterion decision rule. Data from the experiment, collapsed across subjects, are in the left column. Comparable simulated distributions from the Markovian observer are in the right column. Note that zero violations were obtained for the Markovian observer in the $p=s=0.5$ condition. The abscissa is in $z$-score units, in which $\mu_{1}=0$ and $\mu_{2}=1$. The ordinate is in violations per 1,000 trials. 
comparable data generated by the simulated Markovian observer for the actual stimuli received by the subjects. Comparison of simulated and real subjects shows striking similarity of the distributions of violations in the extreme conditions. More modest similarity is seen in the intermediate conditions, where the real subjects do not have as sharp upper and lower cutoffs (the locus of the criteria for the Markovian observer) as does the simulated Markovian observer. Again, in the middle condition, the departure from prediction is dramatic, for no violations can occur for the Markovian observer in that condition. Since the distribution of real subjects' violations is similar in form (although lower and narrower) to those in the other conditions, a simple explanation would be that subjects were continuing to search for and utilize (nonexistent) sequential information in the middle condition, causing violations of the single-criterion rule. Although subjects were somewhat sensitive to the various sequential contingencies, they performed similarly to the Markovian observer only when much sequential information was available. They seem to have persisted in using multiple criteria even when the optimal strategy required a single one. Reasons for this strategy will be discussed later.

Response latencies. Although the subjects were given no speed instruction, the experimental situation contained implicit pressures to complete the session as quickly as possible. Indeed, some subjects showed a tendency to estimate the (constant) interval between feedback presentation and onset of the next trial; "latencies" well below $100 \mathrm{msec}$ were observed for these subjects on a few trials in some conditions. Therefore, we analyzed the response latencies for these data, although some caution must be taken in their interpretation. Figure 2 displays the results, aggregated across subjects and trials. Notice that the horizontal axis portrays $q=r=$ probability of a change of state. The two inner bars in each group of four represent responses consistent with such a change of state; the two outer bars represent responses consistent with no change of state.

The most interesting aspect of the latency data is a tendency for judgments that unlikely transitions had occurred to be accompanied by long latencies. Latencies on such trials tended to be considerably longer than judgments of likely transitions for all subjects. However, because of the nature of the transition matrices governing the sequence of stimuli in the extreme conditions, trials on which such unlikely transitions occurred were few. Thus, there were many more short-latency than long-latency trials, indicating that some caution must be taken in interpreting these latency differences. Nonetheless, the finding is provocative. The present models do not address such data. Unfortunately, models that do address latencies within the signal detection context are designed to explain such effects for sensory stimuli only (e.g., Link, 1975). Since the present stimuli are numerical, the present effects must arise from decision mechanisms, and not from the buildup of sensation or sensory memory.

\section{THE IGNORANT MARKOVIAN OBSERVER}

It is perhaps unfair to condemn the Markovian observer for not being able to predict violations of the singlecriterion rule when $p=s=0.5$. After all, the version

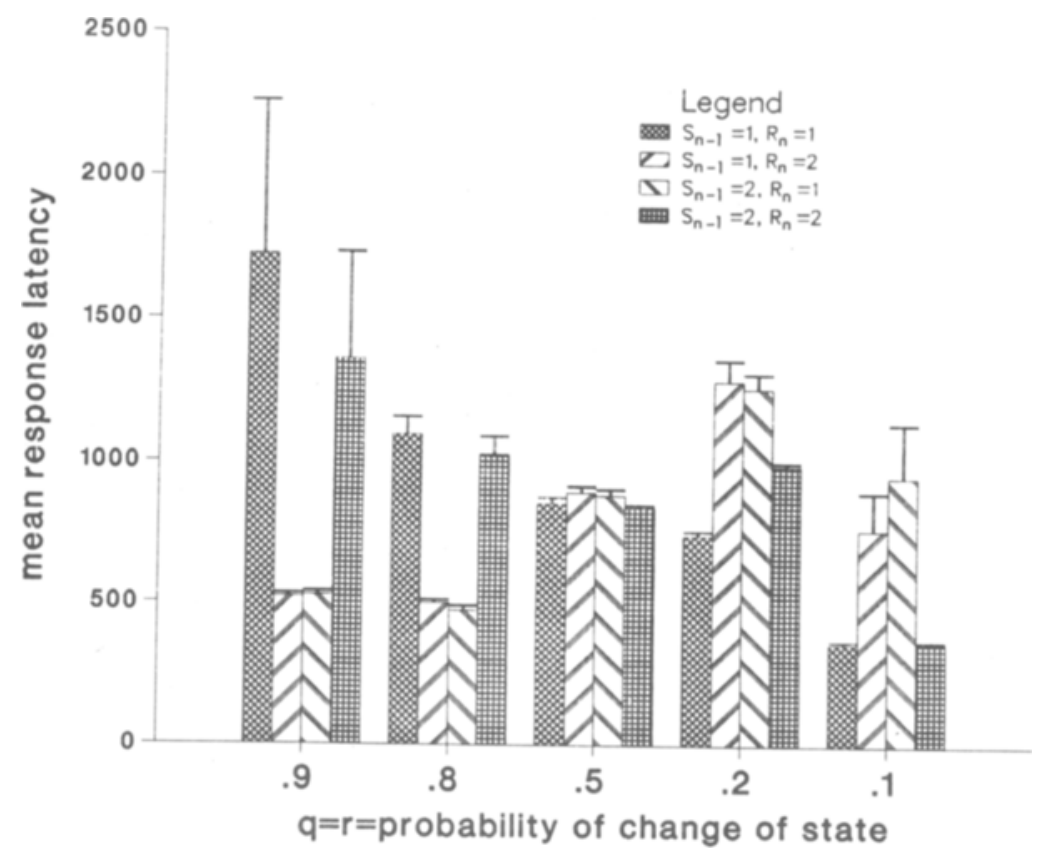

Figure 2. Mean response latency for each combination of response and preceding stimulus state versus $q=r=$ probability of change of state. Error bars are $\pm 1 S E$. 
THE MARKOVIAN OBSERVER

of the model that failed knew everything about the distributions, and the subjects who generated the data did not. Perhaps the departures of subjects' performance from the predictions of the Markovian observer model resulted from the subjects' limited knowledge of the stimulus transition probabilities. To test this notion, we simulated an " 'ignorant" Markovian observer who had to estimate the transition matrix entries from the sequence of stimuli and feedback experienced. The simulation followed the ideallearner model of Kubovy and Healy (1977), except that our ideal learner knew the means of the two distributions, but not the transition matrix, just as did our subjects. Their ideal learner knew the transition matrix (at least that $p=r$ ) but not the means.

The technical details of the simulation will not be presented here. A copy of the computer program used, written in Fortran IV and run on a PDP-11/34 computer, is available from the first author. Basically the simulation implemented the Markovian decision rule, as had the earlier simulation, except that here another part of the program generated samples from one or the other of the two probability distributions in use according to the transition matrix in effect for that run. In addition, instead of using the nominal values of $p, q, r$, and $s$ to calculate $\beta_{1}$ and $\beta_{2}$, the program estimated them from the previous sequence of trials according to $\hat{p}_{k}=k_{11} / k, \hat{q}_{k}=1-\hat{p}_{k}$, $\hat{s}_{k}=k_{22} / k$, and $\hat{r}_{k}=1-\hat{s}_{k}$, where $k_{11}$ is the number of occurrences, in the previous $k$ trials, of the $S_{n-1}=1, S_{n}$ $=1$ transition, and $k_{22}$ is the number of occurrences of the $S_{n-1}=2, S_{n}=2$ transition in the previous $k$ trials. $V_{p m}$ was set equal to 1 for all of the simulations, as it was in the experiment, so that $\beta_{1}$ and $\beta_{2}$ depended only on the estimates of the transition probabilities. To account for the fact that subjects' memories are limited, especially in this experiment, where the stimuli and feedback were presented on video monitors so that the previous sequence of stimuli and feedback was available only through memory, simulations were run for $k$ equal to $5,10,15$, 18,25 , and 50 , as well as the ideal learner (where $k$ was equal to the number of previous trials completed, e.g., $k=$ all). Simulation runs were 1,000 trials in length, and several simulations of this length were run for each value of $p=s$ between 0.1 and 0.9 in steps of 0.1 and for each value of $k$.

The average values of $d^{\prime}$ and $P_{v}$ for 101,000 -trial runs are displayed in Tables 4 and 5 , and the distributions of violations of the single-criterion rule for the same runs are displayed in Figure 3 for $p=s=0.1,0.2,0.5,0.8$, 0.9 , and for $k=15$ and $k=$ all. Several interesting trends are evident in the simulation results. First, notice that the simulations for $k=$ all (the ideal learner) approach the nominal values of $d^{\prime}$ and $P_{v}$ listed in Tables 2 and 3 , with the exception of the $p=s=0.5$ condition, where even the ideal learner violates the singlecriterion rule, although significantly less than do the subjects. Second, Tables 4 and 5 make it clear that the number of trials on which the estimates of $p, q, r$, and $s$ are based $(k)$ drastically affects the performance of the model. For $k=50$, the model performs similarly to the ideal-
Table 4

$d^{\prime}$ for the Simulated Ignorant Markovian Observer

\begin{tabular}{cccccccc}
\hline & \multicolumn{6}{c}{ Basis for Estimates of Transition Probabilities, $\boldsymbol{k}$} \\
\cline { 2 - 8 }$p=s$ & 5 & 10 & 15 & 20 & 25 & 50 & All \\
\hline 0.1 & 2.08 & 2.40 & 2.43 & 2.45 & 2.50 & 2.52 & 2.54 \\
0.2 & 1.25 & 1.55 & 1.64 & 1.70 & 1.71 & 1.75 & 1.78 \\
0.3 & 0.75 & 1.02 & 1.13 & 1.15 & 1.19 & 1.24 & 1.28 \\
0.4 & 0.50 & 0.77 & 0.87 & 0.95 & 0.97 & 1.01 & 1.10 \\
0.5 & 0.46 & 0.65 & 0.74 & 0.79 & 0.84 & 0.89 & 0.96 \\
0.6 & 0.49 & 0.70 & 0.81 & 0.86 & 0.91 & 0.98 & 1.05 \\
0.7 & 0.76 & 1.00 & 1.06 & 1.11 & 1.17 & 1.25 & 1.32 \\
0.8 & 1.21 & 1.41 & 1.51 & 1.59 & 1.64 & 1.72 & 1.76 \\
0.9 & 1.99 & 2.16 & 2.26 & 2.34 & 2.39 & 2.53 & 2.59 \\
\hline
\end{tabular}

Note-Means of 101,000 -trial runs.

learner version, although the number of violations in the $p=s=0.5$ condition increases dramatically. As $k$ decreases, the model performs less like the ideal learner, especially in the middle conditions. At $k=5$ there is little difference in $P_{v}$ across the different values of $p$ (and $s$ ), because of the instability of the estimates of the transition probabilities there. This instability causes $d^{\prime}$ to be much less than optimal at every value of $p=s$, although $d^{\prime}$ is still increased over that attainable by the usual expected-value observer in the extreme conditions. Finally, it will be noticed that $d^{\prime}$ s are lower and $P_{v}$ s higher in the lower left corners of Tables 4 and 5 . This is because estimates of small values of $p=s(0.1$ to 0.3$)$ will be better than those for larger values $(0.7$ to 0.9$)$ for small values of $k$. Inspection of Tables 4 and 5 indicates that a value of $k$ in the vicinity of 15 (or perhaps as high as 50 , if we ignore Subject 4's data) gives an adequate qualitative fit for the average data from the experiment. Here we are balancing the tendency of the $d^{\prime}$ data to be better fit by smaller values of $k$ and the $P_{v}$ data to be better fit by larger values. Also, there is no requirement that subjects must use the same value of $k$ in all conditions. Indeed, there is a suggestion that higher values of $k$ are better for the more extreme conditions. Figure 3 confirms that 15 is a reasonable compromise value for $k$, since the distributions of violations for that value, especially for the middle condition, closely resemble those of the subjects.

Different values of $k$ seem to characterize different subjects' data. Inspection of Tables 2 through 5 together reveals that some subjects seem to perform more like the ideal

Table 5

$P_{v}$ for the Simulated Ignorant Markovian Observer

\begin{tabular}{cccccccc}
\hline & \multicolumn{6}{c}{ Basis for Estimates of Transition Probabilities, $\boldsymbol{k}$} \\
\cline { 2 - 8 }$p=s$ & 5 & 10 & 15 & 20 & 25 & 50 & All \\
\hline 0.1 & .297 & .316 & .324 & .330 & .334 & .339 & .344 \\
0.2 & .279 & .287 & .296 & .301 & .307 & .318 & .329 \\
0.3 & .279 & .254 & .244 & .242 & .241 & .239 & .247 \\
0.4 & .289 & .236 & .210 & .194 & .181 & .154 & .132 \\
0.5 & .304 & .244 & .210 & .181 & .160 & .116 & .042 \\
0.6 & .320 & .262 & .224 & .204 & .188 & .153 & .130 \\
0.7 & .348 & .304 & .280 & .265 & .255 & .248 & .245 \\
0.8 & .356 & .339 & .324 & .320 & .316 & .319 & .327 \\
0.9 & .356 & .342 & .344 & .341 & .339 & .337 & .343 \\
\hline
\end{tabular}

Note-Means of 10 1,000-trial runs. 

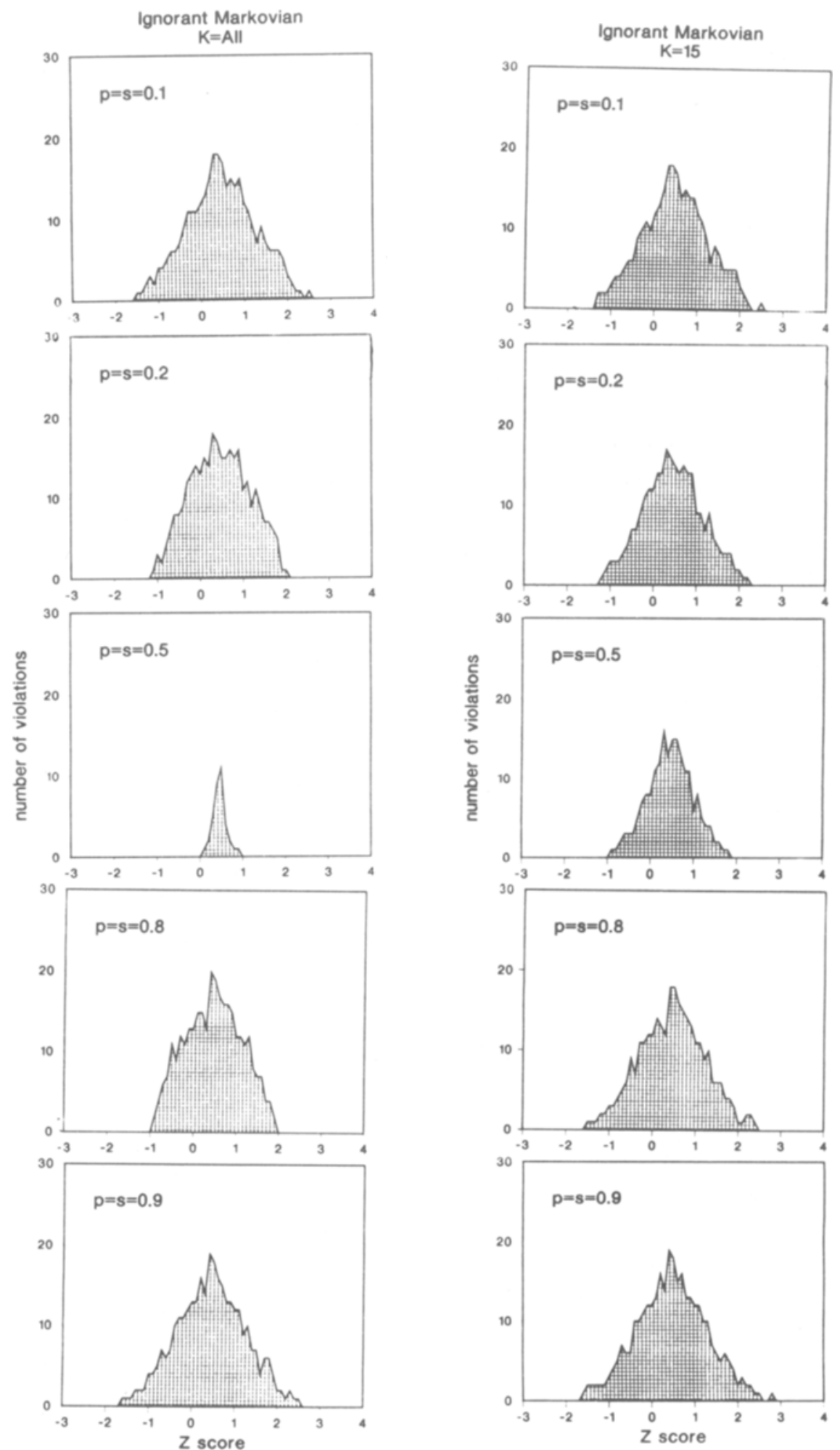

Figure 3. Distributions of violations of the single-criterion decision rule for the simulated ignorant Markovian observer. Left column: $k=$ all; right column: $k=15$, where $k$ is the basis for estimating $p, q, r$, and $s$. The distributions are based on 101,000 -trial simulation runs in each condition. The abscissa is in $z$-score units, in which $\mu_{1}=0$ and $\mu_{2}=1$. Ordinate is in violations per 1,000 trials. 
learner (e.g., Subject 1) and others more like a Markovian observer with a somewhat limited memory (e.g., Subject 3 ). Therefore, the single parameter, $k$, can also account for some of the individual differences apparent in the data. The clearly aberrant Subject 4 , who actually violated the single-criterion rule most in the middle condition, seems to have been operating according to a rule that ignored the distributional information available. His data could be explained by a tendency to simply respond with the most likely distribution (based on estimates of the transition probabilities in force) regardless of the sample value. This strategy would more closely resemble the Markovian observer, the more extreme $p$ and $s$ were, and this subject's data show this pattern. Moreover, most of the violations of the single-criterion rule in the middle condition that were far removed from that criterion came from this subject. Therefore, the other 4 subjects' data are even better explained by the ignorant Markovian simulations for this condition when this is taken into account.

One remaining problem with the models developed so far is that they require the same decision process on each trial. Therefore, they cannot make predictions of different response latencies conditional on previous trials. However, response latencies in this task are conditional on previous trials. Response latencies were longest for judgments that an unlikely transition had occurred. This suggests that subjects were prepared to make a response consistent with the transition matrix governing the sequence of samples, and hesitated to make a response when stimulus information was inconsistent with that matrix.

To explain these latency data, we have to modify the decision process used by the ignorant Markovian. The simplest way to do this is to assume that the response judged most likely according to the estimated transition probabilities and the immediately previous distribution sampled is prepared. Then, when the next sample is presented, if the relative likelihood information is consistent with the prepared response, that response is given; if not, then the other response is given, after a short delay caused by either rechecking or preparation of the other response, or both. A somewhat less palatable possibility is that on some trials responses are based purely on sequence information, whereas on other trials distributional information is used. One possibility would be to use the values of $\beta_{1}$ and $\beta_{2}$ estimated from the previous sequence of samples as criterion-band boundaries, making decisions based on sequence information whenever a sample value fell within the band, and basing decisions on distributional information whenever a sample value fell outside the band. If, as above, the response consistent with sequence information were prepared, and if the sample fell within the criterion band, then such responses would be, on the average, the fastest. Responses based on distributional information, some of which would be inconsistent with sequence information, would be slower. Thus, "most likely" responses would be a combination of the faster responses to samples within the criterion band and the slower ones to samples outside it, whereas the "least likely" responses would be made up only of the slower response to samples outside the criterion band. This would make the model very similar to previously proposed criterion-band models (Parducci \& Sandusky, 1965; Sandusky, 1971; Ward, 1973), but with the improvement that a reasonable basis for setting the band boundaries is provided. The present data are probably not adequate to differentiate these and other possible models that incorporate latency mechanisms. However, they do suggest that such models should be developed.

\section{THE COMPLETELY IGNORANT MARKOVIAN OBSERVER}

As developed above, the ignorant Markovian observer can be readily generalized to situations involving probabilistic categorization of sensory stimuli. Such a generalization would involve making assumptions about the distributions from which the $X_{n}$ are sampled similar to those made in signal detection theory. However, observers in such situations are typically even more ignorant than the Markovian observer developed above. For example, as assumed by Kubovy and Healy (1977), they typically do not know (and probably in principle cannot know) either the means or the standard deviations of the distributions from which stimuli are sampled. Thus, they would have to estimate them from the sequence of stimuli experienced in the experiment. Presumably the same problems would arise with such estimates as those discussed above for estimates of the transition probabilities. Also, since so many estimates would have to be made, problems of information overload might arise, and would be solved by using heuristics to accomplish the estimates. Obviously, studies of such situations and such models remain to be done.

Another generalization of the present models would be to no-feedback situations. The simplest model for such situations would have the observer use the previous response, $R_{n-1}$, as an estimate of $S_{n-1}$, and make the required estimates of transition probabilities, means, and standard deviations as usual. This would introduce additional variability, and perhaps additional biases as well, into the estimates. Such variability, and bias magnitude, would be negatively correlated with $d^{\prime}$. Again, studies of such a generalization remain to be done.

Finally, there are a variety of other influences on the criteria in such situations, for example, Treisman and Williams's (1984) stabilization and tracking adjustments, those of the payoff matrix in force, the gamblers' fallacy, and directional stimulus generalization. Some of these may turn out to be incorporated in the estimation procedures of the completely ignorant Markovian observer. For example, Treisman and Williams's tracking system operates similarly to the ignorant Markovian observer's adjustments of criteria dependent on the most recent relative likelihoods of the stimulus distributions. If, say, distri- 
bution 2 has occurred more often in recent trials, criteria will be adjusted downwards in order to increase the likelihood of making the corresponding response.

On the other hand, other such systems of criterion adjustment seem not to be accounted for by the existing model. In the cases of stabilization, gamblers' fallacy, or directional stimulus generalization, additional parameters can be easily added to the ignorant Markovian model to produce the desired effects. Although ad hoc at present, such additions should allow testable predictions to be made about the interactions of the various biases.

\section{REFERENCES}

ANDERSON, N. H. (1960). Effect of first-order conditional probability in a two-choice learning situation. Journal of Experimental Psychology, 59, 73-93.

EsTES, W. K. (1972). Research and theory on the learning of probabilities. Journal of the American Statistical Association, 67, 81-102.

Green, D. M., Swets, J. A. (1974). Signal detection theory and psychophysics. New York: Krieger. (Reprint of 1966 edition)

Kubovy, M., \& Healy, A. (1977). The decision rule in probabilistic categorization: What it is and how it is learned. Joumal of Experimental Psychology: General, 106, 427-446.

Kubovy, M., RAPOPORT, A., \& TVersky, A. (1971). Deterministic vs. probabilistic strategies in detection. Perception \& Psychophysics, 9 , $427-429$.

LARKIN, W. (1971). Response mechanisms in detection experiments. Joumal of Experimental Psychology, 91, 140-153.
LEE, W. (1971). Decision theory and human behavior. New York: Wiley.

LiNk, S. W. (1975). The relative judgment theory of two-choice response time. Journal of Mathematical Psychology, 12, 114-135.

Parducci, A., \& SANDUSKY, A. (1965). Distribution and sequence effects in judgment. Journal of Experimental Psychology, 69, 450-459.

RestLe, F. (1966). Run structure and probability learning: Disproof of Restle's model. Journal of Experimental Psychology, 72, 382-389.

Rose, R. M., \& VITZ, P. C. (1966). The role of runs of events in probability learning. Journal of Experimental Psychology, 72, 751-760.

SANDUSKy, A. (1971). Signal recognition models compared for random and Markov presentation sequences. Perception \& Psychophysics, 10, 339-347.

SChOEFFLER, M. S. (1965). Theory for psychophysical learning. Journal of the Acoustical Sociery of America, 37, 1124-1133.

Thurstone, L. L. (1927). A law of comparative judgment. Psychological Review, 34, 273-286.

Treisman, M., \& Williams, T. C. (1984). A theory of criterion setting with an application to sequential dependencies. Psychological Review, 91, 68-111.

VITz, P. C., \& ToDD, T. C. (1967). A model of learning for simple repeating binary patterns. Journal of Experimental Psychology, 75, 108-117.

WARD, L. M. (1973). Use of Markov-encoded sequential information in numerical signal detection. Perception \& Psychophysics, 14, 337-342.

(Manuscript received February 16, 1987; revision accepted for publication July 20,1987 , 